\title{
Human embryonic stem cells contribute to embryonic and extraembryonic lineages in mouse embryos upon inhibition of apoptosis
}

Cell Research (2018) 28:126-129. doi:10.1038/cr.2017.138; published online 3 November 2017

\section{Dear Editor,}

Recently, interspecies chimera formation has been established in rodents by injection of rat pluripotent stem cells (PSCs) into mouse early embryos, and such a system provides an in vivo assay to test the developmental potential of human PSCs (hPSCs) [1]. In addition, the interspecies chimeras formed between hPSCs and large animal embryos would open new avenues to generate human tissues and organs for regenerative medicine [2]. In rodents, embryonic stem cells (ESCs) and epiblast stem cells (EpiSCs) have been derived from inner cell mass (ICM) of the blastocyst and post-implantation epiblast respectively $[3,4]$. Although both being pluripotent, the ESCs and EpiSCs are considered to represent two distinct states of pluripotency, the naïve and primed pluripotent state. Only naïve state ESCs can colonize the early embryos before the blastocyst stage to form adult chimeras and transmit to the germline, while the primed state EpiSCs can only integrate into the post-implantation embryos $[5,6]$. Intriguingly, although derived from the ICM, the human ESCs (hESCs) are similar to the mouse EpiSCs in many aspects such as the morphology and self-renewal pathways, hence they are considered to represent the primed pluripotent state. Several studies have reported the generation of human-animal interspecies chimeras using the stage-matching hESCs. For example, primed hPSCs were engrafted into in vitro cultured gastrula-stage mouse embryos to form chimeras, and the primed hPSCs were also converted into a naïvelike state and then were injected into pre-implantation embryos to form mouse or pig chimeras [7-9]. Recently, a non-stage-matching approach has been established to generate mouse-rat interspecies chimeras by inhibiting apoptosis of the primed ESCs [10]. We hence hypothesize that inhibition of apoptosis may enable the primed $\mathrm{hESCs}$ to form interspecies chimeras upon injection into mouse pre-implantation stage embryos.

We used a doxycycline (DOX)-inducible system for transient induction of human anti-apoptotic genes, $B C L 2 L 1$ or $B C L 2$, together with the DsRed reporter gene, in hESCs carrying a constitutively expressed reporter gene GFP (Supplementary information, Figure S1A and S1B). The $B C L 2 L 1$ - and $B C L 2$-overexpressing hESCs maintained their pluripotent state and could differentiate into embryoid bodies (EBs) expressing marker genes of all three germ layers (Supplementary information, Figure S1C-S1F). Compared with the control hESCs, the $B C L 2 L 1$ - and $B C L 2$-overexpressing hESCs displayed much lower levels of spontaneous apoptosis and significantly higher single cell cloning efficiency (Supplementary information, Figure S1G and Figure 1A). Global gene expression profiles revealed that $B C L 2 L 1$ and $B C L 2$ overexpression had little effect on the hESC pluripotent state, as only 40 and 48 differentially expressed genes (DEGs) were identified in the BCL2L1- and BCL2-overexpressing hESCs respectively, which were involved in functions related to cellular response to DNA damage stimuli and extracellular stimuli, and regulation of apoptotic process (Supplementary information, Figure S1H$\mathrm{S} 1 \mathrm{~K})$. Consistently, BCL2L1- and BCL2-overexpressing hESCs were clustered with the primed state hESCs but not with the previously reported naïve state hESCs by principal component analysis [9], and did not upregulate naïve marker gene expression (Supplementary information, Figure S1L and S1M). Taken together, these results suggest that $B C L 2 L 1$ or $B C L 2$ overexpression can inhibit apoptosis in hESCs without affecting their pluripotent state.

Next, we investigated whether the inhibition of apoptosis could enable the hESCs to colonize early stage mouse embryos. A total of 76 blastocysts were generated from 89 4-cell embryos injected with $B C L 2 L 1$-overexpressing hESCs, and 72 of them ( $95 \%)$ contained GFP-positive human cells. In contrast, GFP fluorescence was scarcely observed in the blastocysts injected with control hESCs due to apoptosis (Supplementary information, Figure S2A, S2B and Table S1, S2). Intriguingly, 
A

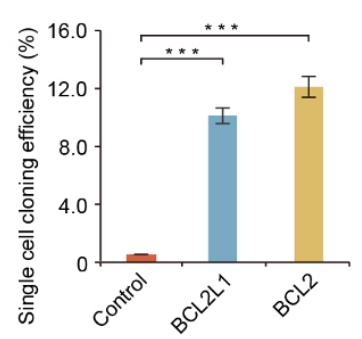

B

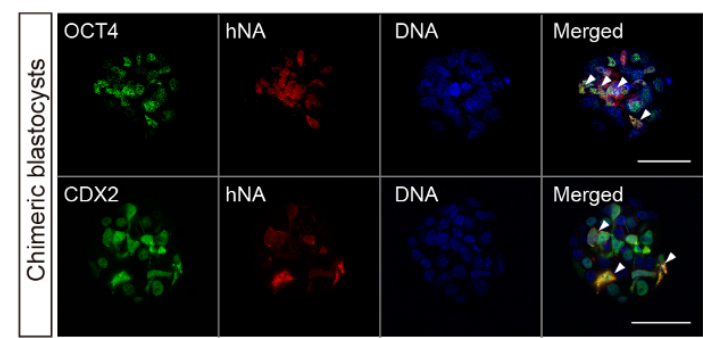

C

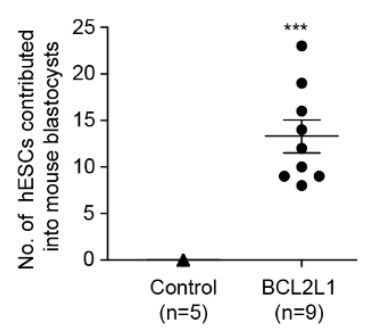

D

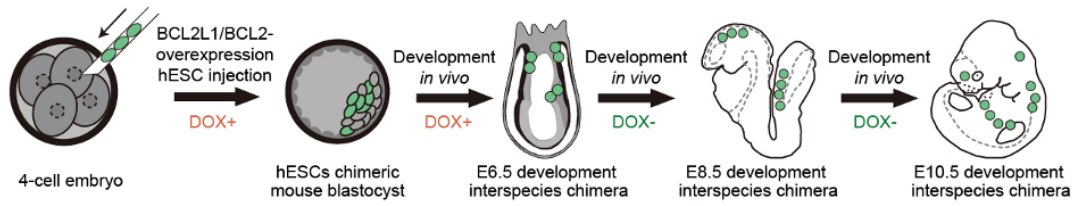

E

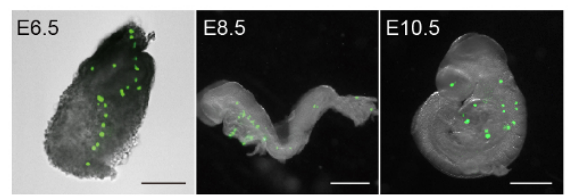

$\mathrm{F}$

SOX2 / hNA / DNA
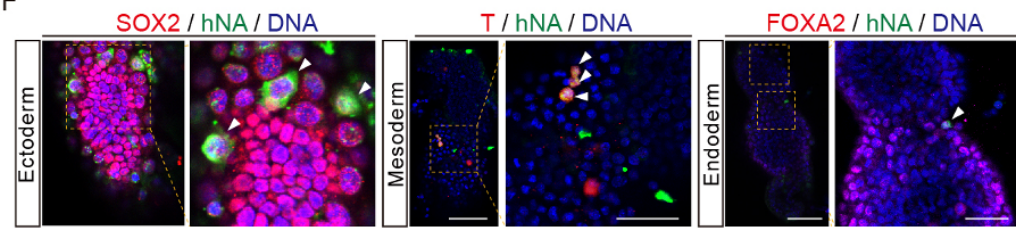

$\mathrm{H}$

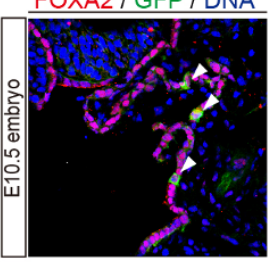

$J$

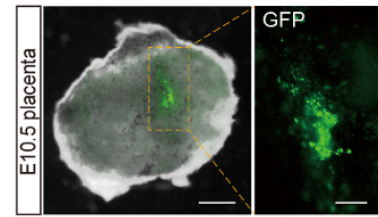

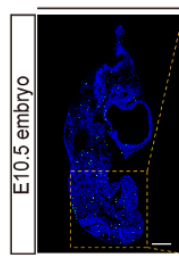

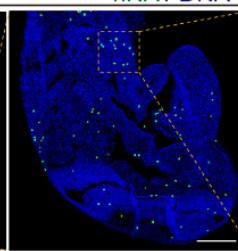

$\mathrm{K}$

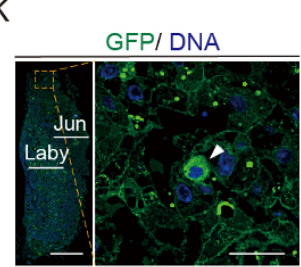

$\mathrm{L}$

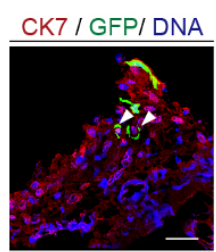

I

\begin{tabular}{|c|c|c|c|c|}
\hline \multirow{2}{*}{$\begin{array}{c}\text { Donor cell lines } \\
\text { (nESCs) }\end{array}$} & \multirow{2}{*}{$\begin{array}{l}\text { Embryos } \\
\text { transferred }\end{array}$} & E6.5 & E8.5 & E10.5 \\
\hline & & \multicolumn{3}{|c|}{ Chimerasspups (\%) Chimerasspups (\%) Chimeras $/$ pups (\%) } \\
\hline BCLLL1-1 & 130 & $\begin{array}{l}36.45 \\
(80.0)\end{array}$ & - & \\
\hline BCLLL1-2 & 81 & - & $\begin{array}{c}7 / 16 \\
(43.8)\end{array}$ & \\
\hline BCL2L1-3 & 70 & - & & $\begin{array}{l}12 / 22 \\
(54.5)\end{array}$ \\
\hline BCL2-1 & 30 & $\begin{array}{l}12116 \\
(75.0)\end{array}$ & - & - \\
\hline BCL2-2 & 79 & - & $\begin{array}{l}13 / 35 \\
(37.1)\end{array}$ & - \\
\hline BCL2-2 & 132 & - & - & $\begin{array}{l}5 / 13 \\
(38.5)\end{array}$ \\
\hline
\end{tabular}

*BCL2L1-1 hESCs without DOX treatment were used as control. $\mathrm{M}$

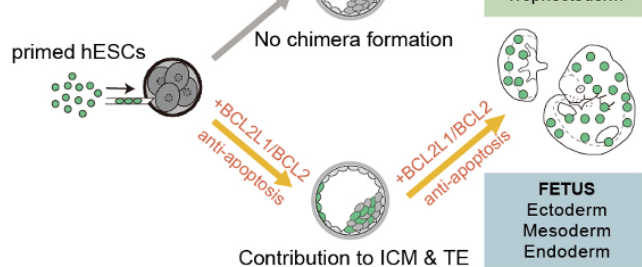

Figure 1 Overexpression of anti-apoptotic genes enables hESCs to differentiate into embryonic and extraembryonic lineages in pre- and post-implantation mouse embryos. (A) Colony formation efficiency of the BCL2L1- and BCL2-overexpressing hESCs. The wild-type hESCs were used as control. Data are displayed as mean \pm SEM of three biological replicates. ${ }^{* * *} P<0.001$. (B) Immunofluorescent staining of OCT4 (specific marker of the ICM lineage), CDX2 (specific marker of the TE lineage) and the human nuclear antigen (hNA) in chimeric blastocysts of BCL2L1-overexpressing hESCs and mouse early embryos. White arrowheads indicate OCT4 and hNA double-positive cells, and CDX2 and hNA double-positive cells respectively. Scale bars, $100 \mu \mathrm{m}$. (C) Quantitative analysis of the number of hNA-positive hESC-derived cells integrated into interspecies chimeric mouse blastocysts. BCL2L1 transgenic hESCs without DOX treatment were used as control. Data are displayed as mean \pm SEM of three biological replicates. ${ }^{* * *} P<0.001$. (D) Schematic overview of the strategy to generate post-implantation interspecies chimeras by injecting $B C L 2 L 1 / B C L 2$-overexpressing hESCs into 4-cell stage mouse embryos. (E) Representative phase contrast images of E6.5, E8.5 and E10.5 mouse chimeric embryos containing GFP-positive hESC-derived cells. Scale bars, $150 \mu \mathrm{m}$ (left), 200 $\mu \mathrm{m}$ (middle), $1 \mathrm{~mm}$ (right). (F) Immunofluorescent staining of ectoderm-specific marker SOX2 (left panel), mesoderm-specific marker T (Brachyury) (middle panel), and endoderm-specific marker FOXA2 (right panel) in human-mouse interspecies chimeric embryos at E6.5. hNA was used for detecting hESC-derived cells. Scale bars, $100 \mu \mathrm{m}$ (left) and $50 \mu \mathrm{m}$ (right) for each panel. (G) Immunofluorescent staining of FOXA2 in E10.5 interspecies chimeric embryos. hNA staining was used for detecting hESC-derived cells. White arrowheads indicate FOXA2 and GFP double-positive cells. (H) Immunofluorescent staining of hNA for detec- 


\section{Wei $\mathrm{Li}^{1,2}$, Baoyang $\mathrm{Hu}^{1,2}$}

${ }^{I}$ State Key Laboratory of Stem Cell and Reproductive Biology, Institute of Zoology, Chinese Academy of Sciences, Beijing 100101, China, ${ }^{2}$ University of Chinese Academy of Sciences, Beijing 100049, China; ${ }^{3}$ College of Life Science, Northeast Agricultural University of China, Harbin, Heilongjiang 150030, China

*These five authors contributed equally to this work.

Correspondence: Baoyang $\mathrm{Hu}^{\mathrm{a}}$, Wei $\mathrm{Li}^{\mathrm{b}}$, Qi Zhou ${ }^{\mathrm{c}}$

âE-mail: byhu@ioz.ac.cn;

bE-mail: liwei@ioz.ac.cn;

'E-mail: qzhou@ioz.ac.cn

\section{Reference}

1 Kobayashi T, Yamaguchi T, Hamanaka S, et al. Cell 2010; 142:787799.

2 Wu J, Greely HT, Jaenisch R, et al. Nature 2016; 540:51-59.

3 Brons IG, Smithers LE, Trotter MW, et al. Nature 2007; 448:191-195.

4 Ying QL, Wray J, Nichols J, et al. Nature 2008; 453:519-523.

5 Huang Y, Osorno R, Tsakiridis A, et al. Cell Rep 2012; 2:1571-1578.

6 Koller BH, Smithies O. Annu Rev Immunol 1992; 10:705-730.
7 Mascetti VL, Pedersen RA. Cell Stem Cell 2016; 18:67-72.

8 Theunissen TW, Friedli M, He Y, et al. Cell Stem Cell 2016; 19:502515.

9 Wu J, Platero-Luengo A, Sakurai M, et al. Cell 2017; 168:473-486. e415.

10 Masaki H, Kato-Itoh M, Takahashi Y, et al. Cell Stem Cell 2016; 19:587-592.

(Supplementary information is linked to the online version of the paper on the Cell Research website.)

This work is licensed under a Creative Commons Attribution 4.0 Unported License. The images or other third party material in this article are included in the article's Creative Commons license, unless indicated otherwise in the credit line; if the material is not included under the Creative Commons license, users will need to obtain permission from the license holder to reproduce the material. To view a copy of this license, visit http:// creativecommons.org/licenses/by/4.0/

(C) The Author(s) 2017 